\section{A Milano dopo Citylife e Porta Nuova: ancora sparkling buildings contro town planning?}

Sergio Brenna
Politecnico di Milano, Dipartimento di Architettura e Studi Urbani (sergio.brenna@polimi.it)

Un bilancio degli esiti delle trasformazioni urbane dell'ultimo quindicennio nelle aree più centrali di Milano stenta ancora a recepire un ragionamento non fondato unicamente sul condizionamento del momentaneo favore suscitato dal successo di pubblico riscosso dall'effimera novità di immagine (che pure si deve riconoscere esservi, ma forse a seguito di un troppo lungo periodo di inerte incapacità a produrre cambiamenti pensati dello spazio pubblico urbano, e che quindi può esservi indipendentemente dalla bontà e condivisione del risultato) conseguita nel breve periodo dagli edifici realizzati nell'ambito dei Programmi Integrati di Intervento noti come Porta Nuova e Citylife. In forza del successo di pubblico che quei contesti, realizzandosi, vanno riscuotendo soprattutto nella popolazione che è ormai in uso chiamare metrolife o happy hour style per la giocosa attrattiva della sgargiante luminosità delle ondulate trasparenze vetrate degli edifici e degli zampillanti giochi d'acqua colorati, ciò che - a detta dei fautori di quelle soluzioni di immagine - avrebbe definitivamente sconfitto e messo a tacere le critiche di quanti si erano opposti agli esiti di quei progetti di assetto urbano. Sviluppo in altezza, inusualità di forme e materiali e autonoma istantaneità del linguaggio espressivo-progettuale (talvolta connotato come 'sparkling' o 'smart') e innovative potenzialità tecnologiche nell'autonomia energetica e nel contenimento di emissione di rifiuti hanno preteso di giustificare il progressivo totale isolamento tra edificio e contesto urbano.

Il dibattito si attarda, quindi, in un clima di compiaciuta autocelebrazione degli effimeri successi di immagine e di pubblico di quegli edifici e dell'evento di Expo 2015, i cui padiglioni sono in corso di rapida demolizione e la cui area, tuttavia stenta particolarmente a trovare un uso e un senso strategico nel dopo-evento, e ci si propone che quei modelli di trasformazione urbana possano essere riprodotti nuovamente nel prossimo futuro come canone per una ancor più vasta estensione di aree, che segnerebbero nel lungo periodo il funzionamento e l'assetto dell'intera città.

L'urbanistica e il town planning, dopo essere stati oggetto di grandi aspettative e rivendicazioni sociali tra gli anni ' 60 e ' 80 , negli ultimi decenni, non godono più di una buona fama in un periodo di difficoltà finanziarie e di rapidità di mutamenti economico-produttivi e il loro posto nell'immaginario sociale collettivo dell'aspettativa di un futuro migliore è stato preso dall'ambientalismo ecologista o dal liberismo da regole insediative per incentivare lo sviluppo economico e immobiliare. uttavia, per non ridurre il dibattito sulla morfologia urbana a
Parole chiave: pianificazione negoziata; VAS; indici di pianificazione 

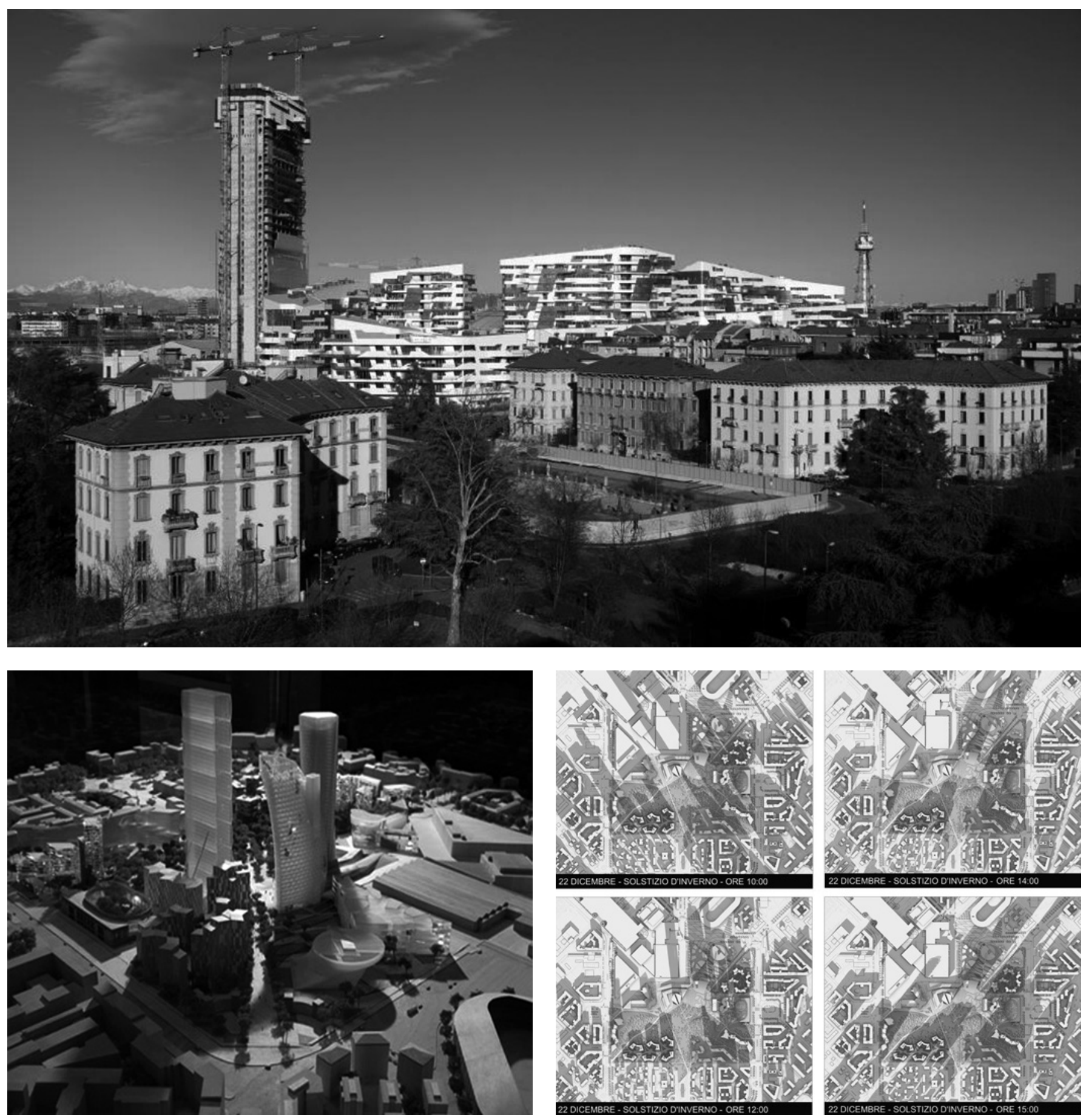

Dall'alto:

- Progetto Citylife in attuazione al 2014: sulla destra gli edifici residenziali di Zaha Hadid e Daniel Libeskind: al centro la torre ad uffici di Arata Isozaki

Fonte: Stefano Gusmeroli, Milanofoto.it

- Progetto Citylife sull'area dell'ex Fiera di Milano, plastico del progetto a cura della società attuatrice, 2009

Fonte: Società Citylife

- Progetto Citylife, studio delle ombre portate al solstizio di inverno, elaborato digitale della società attuatrice ai fini dell'approvazione del Programma Integrato di Intervento (PII) Fonte: Società Citylife-Comune di Milano 
mero pettegolezzo sulle preferenze estetiche personali di questo o quel politico o amministratore pubblico, per quanto volitivo, di questo o quel progettista, per quanto famoso, forse si dovrebbe partire dall'immagine data da un ragionevole rapporto tra edificio, densità di popolazione e quantità edificabile (cioè la densità fondiaria ${ }^{1}$ ) per definirne il conseguente rapporto tra spazi pubblici e privati, anziché partire da una immotivata fissazione della percentuale di superficie pubblica rispetto a quella totale, quale ad esempio il 50\% (arbitrariamente fissato nei più noti e controversi progetti del cosiddetto Rinascimento Urbano a Milano - Citylife, Porta Nuova - e in molti altri casi nel resto d'Italia), tesa solo a stupire il pubblico con l'illusione di una sorta di equa spartizione mezzadrile, invece assolutamente immotivata sul piano logico-razionale del rapporto con gli indici edificatori fissati negozialmente solo sulla base del valore di rendita fondiaria atteso dalla proprietà, abilmente celata dalla capacità di una cultura progettuale dell'immagine mediatica che attiene più al carattere della riconoscibilità del marchio aziendale, del logo pubblicitario, della novità effimera della moda e del design che non all'individuazione di tendenze stabili e durature, meglio confacenti a fenomeni di lunga durata come quelli della città e dell'ambiente.

Insomma, forse sarebbe bene estendere le rivendicazioni 'no logo' già manifestatesi nel campo della moda, del design, della pubblicità anche al campo della pianificazione urbana e manifestazioni della creatività architettonico-progettuale!

Affinché la retorica sulle tecnologie delle 'smart cities' e le procedure per la Valutazione Ambientale Strategica (VAS) non sovrastino coi propri criteri di sostenibilità un ragionevole rapporto tra fattori dell'assetto insediativo, e consentano, quindi, un confronto non condizionato a priori tra le diverse opzioni planivolumetriche possibili, è necessario che le amministrazioni pubbliche fissino indici di edificabilità territoriale e fondiaria e dotazioni di spazi pubblici non negozialmente, ma in modo logicamente coerente con gli obiettivi perseguiti.

È ciò che l'urbanistica e la progettazione urbana più consapevoli dovrebbero rivendicare da una piena integrazione delle procedure di Valutazione Ambientale Strategica (VAS), in passato troppo a lungo ignorate dalla normativa della disciplina urbanistica, la quale però oggi viceversa rischia a sua volta subirne passivamente metodiche riduttive che si dimostrano essere a loro volta inadeguate a governare l'esito della forma urbana.

Ciò è possibile con formule di coerenza ${ }^{2}$ che eguaglino la quantità edificabile generata dagli indici edificatori attribuiti all'area in progetto con quella generata dall'indice di dotazione di area pubblica pro capite che si intende ottenere. Ciò impedirebbe che, persino con le verifiche VAS, le ombre proiettate da edifici alti di una nuova area di pianificazione oscurino per lunghi periodi della giornata, soprattutto durante il periodo invernale, quelli delle zone vicine, come, invece, accade sull'area dell'ex Fiera di Milano con il progetto Citylife: le tre torri di 180-220 $m$ di altezza, consentite dagli elevati $\mathrm{I}_{\mathrm{et}}$ ed $\mathrm{I}_{\mathrm{ef}}$ arbitrariamente negoziati in assenza di vincoli tipologici, nel periodo attorno al solstizio d'inverno generano ombre che oscurano gli edifici circostanti dalle ore 10.00 alle 15.00 , ciò che significa in pratica durante tutta la giornata solare, provocando un deterioramento nella qualità ambientale degli edifici circostanti.

È sostenibile a lungo una tale contraddizione o non occorrerebbe invece una condizione di reciproco rafforzamento delle
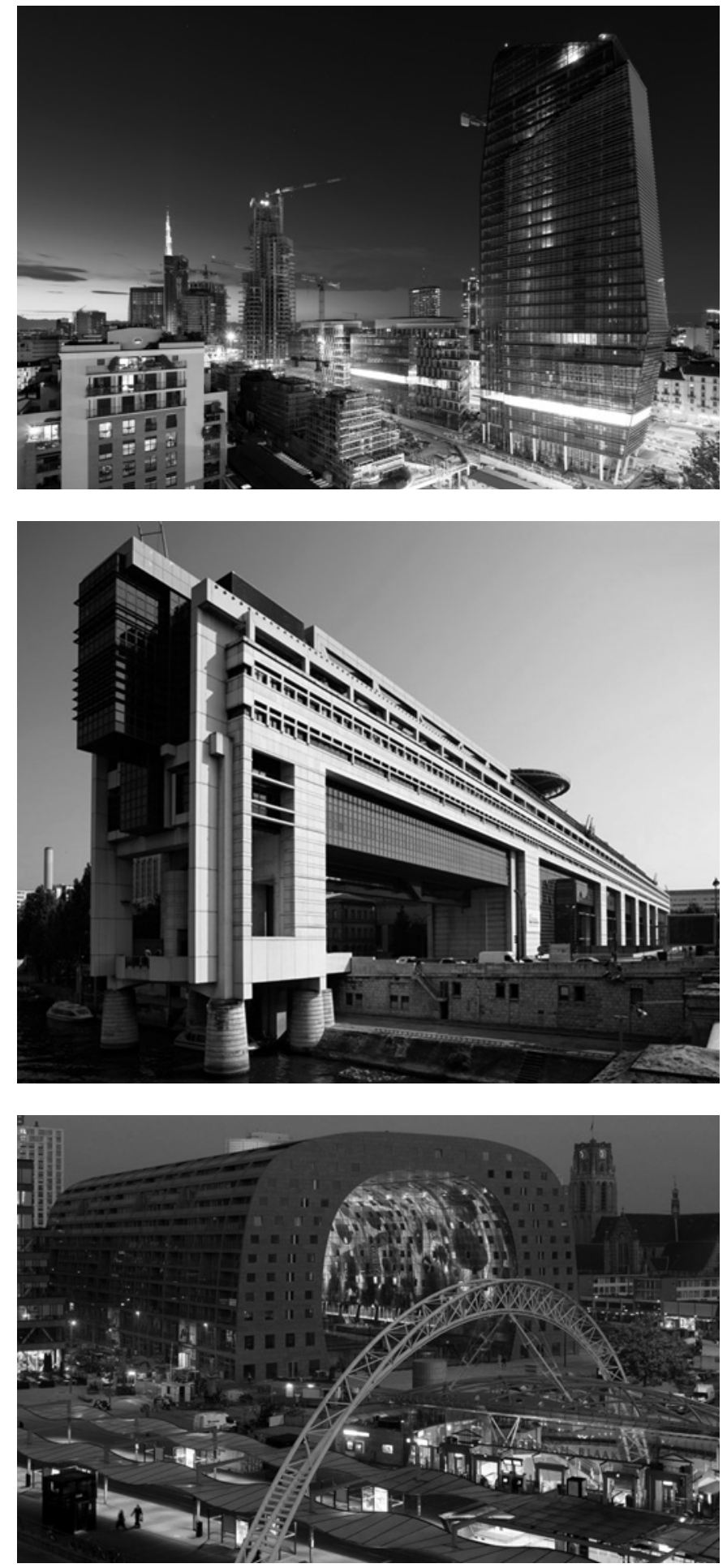

Dall'alto:

- Esito della 'libera' progettualità di s.l.p. e altezza di piano dei $250.000 \mathrm{mc}$ ottenuti per sentenza da parte della proprietà: in primo piano la torre 'Il Diamantone' di Caputo-Kohn-Pedersen- Fox

Fonte: Stefano Gusmeroli, Milanofoto.it

- Chemetov e Huidboro: nuovi uffici del Ministero delle Finanze francese a Parigi-Bercy, 1989

Fonte: Sito web dello Studio Chemetov-Huidboro - Studio MVRDV, Markthal, Rotterdam, vista del fronte, dell'interno e dintorni, 2014

Fonte: sito web del Comune di Rotterdam 

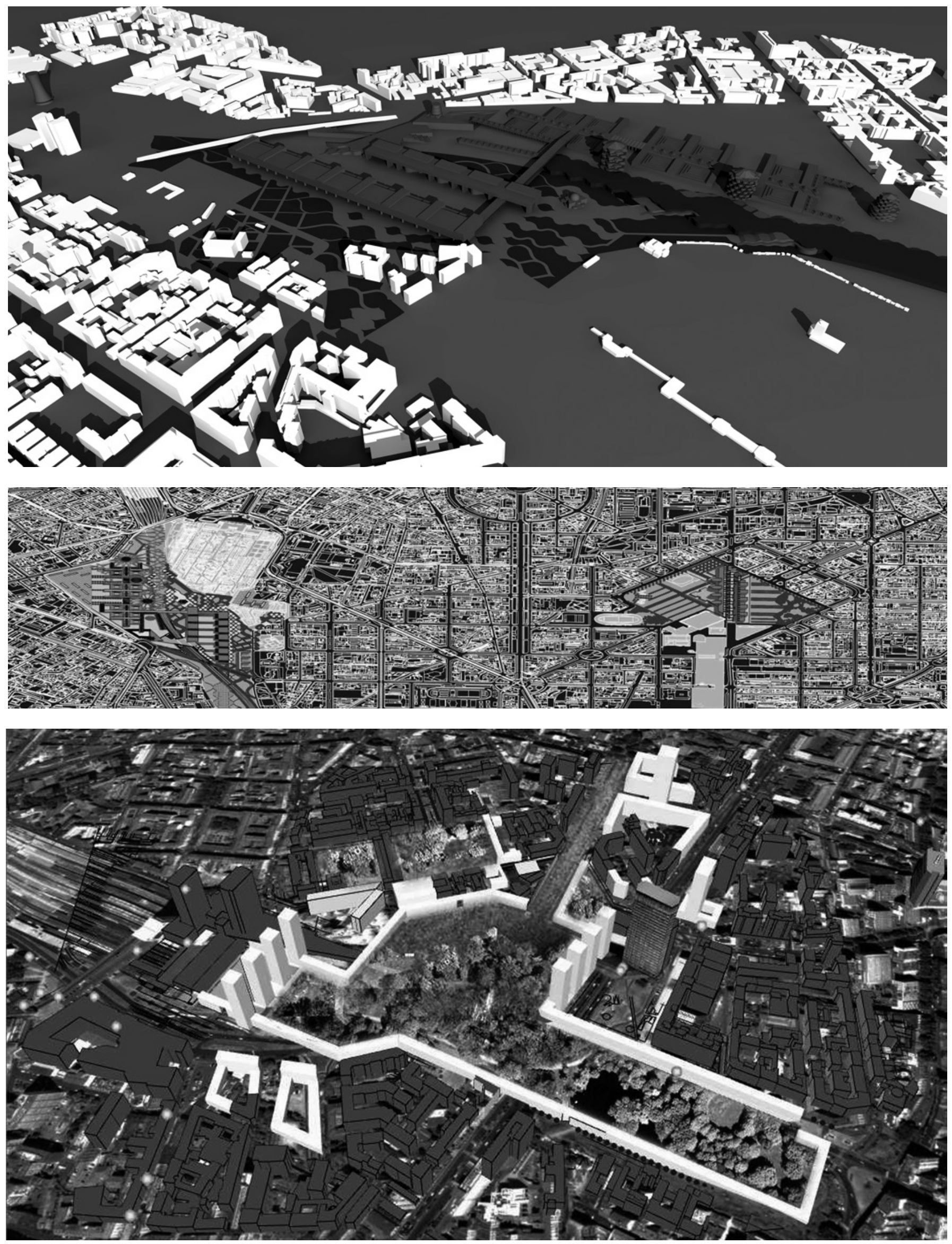

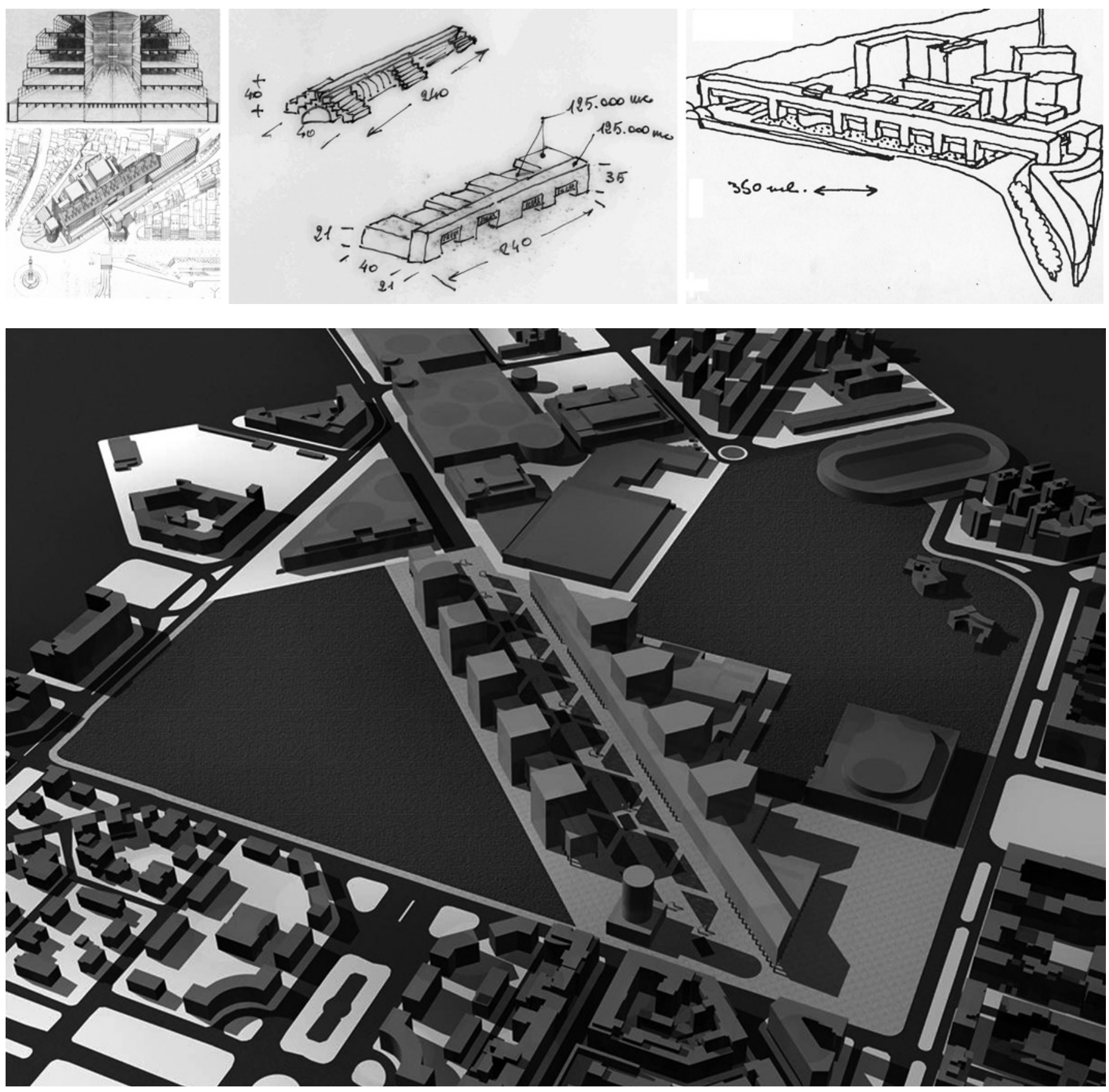

In questa pagina:

- Brenna: soluzioni compatte dei 250.000 mc concessi dal Comune sull'area ex-Varesine per sentenza giudiziaria

(da un progetto inattuato di Guido Canella e da uno attuato di Paul Chemetov), 2008

Fonte: Archivio dell'autore

- Brenna, Enriquez: progetto Strada Lombarda all'ex Fiera di Milano. Rendering da sud-ovest; boulevard pedonale

al centro con edifici porticati ai lati e verde compatto sui fronti esterni

Fonte: Archivio degli autori

Nella pagina precedente, dall'alto:

- Brenna, Enriquez: assetto insediativo di riuso dell'ex scalo ferroviario Farini, con impianto a elementi lineari,

piazze a corte ed edifici a pianta centrale. Workshop progettuale 2009

Fonte: Archivio degli autori

- Brenna, Enriquez: riutilizzo di ex Fiera di Milano e di ex scalo Farini sugli orientamenti della direttrice

nord-ovest di Milano, con proiezioni sull'assetto di Nuova Bovisa ed Expo 2015

Fonte: Archivio degli autori

- Gardella, Sacerdoti: progetto Parco possibile a Porta Nuova alternativo a quello attuato da Hines-Catella,

pur con le medesime quantità edificatorie, 2010

Fonte: Archivio degli autori 
proprie specificità scientifico-disciplinari? Con opportune scelte tipologico-insediative e di compattamento volumetrico - sulla cui attuabilità non possono esistere dubbi avendo già trovato concrete realizzazioni a Parigi e a Rotterdam, pur più vincolanti per la libera fungibilità di mercato (residenza/terziario) ora interamente lasciata alle convenienze tipologico-costruttive dei promotori immobiliari, si attuerebbe nel riuso delle aree lungo la storica direttrice nord-ovest di Milano (ex Fiera Milano, ex CD Garibaldi/Repubblica/Varesine, ex scalo ferroviario Farini, Bovisa, aree del dopo Expo, ecc.) quella coerenza complessiva tra corretti orientamenti urbanistico-insediativi e qualità tecnologica degli edifici ${ }^{3}$, determinante per quel carattere fondativo di effettivo Nuovo Rinascimento Urbano, sinora totalmente assente nei tracciati prodromici attuati da Citylife e Porta Nuova ${ }^{4}$.

Lo spettro di una seconda Porta Nuova sull'ex Scalo Farini, preannunciato nel settembre 2015 dall'AD di FS in occasione della presentazione del Protocollo preliminare d'Intesa con la Giunta milanese ci dice infatti che gli indici edificatori contrattati ancora una volta sono incongruenti (anche se meno di Citylife e Porta Nuova): infatti, pur di far tornare i conti di FS e Comune, accomunati nel lucrare l'aspettativa di rendita fondiaria si contratta un indice di edificabilità territoriale $\mathrm{I}_{\mathrm{t}}=0.70 \mathrm{mq} / \mathrm{mq}$ con cui non si possono ricavare sulle aree FS i $15 \mathrm{mq} / \mathrm{ab}$., di parchi territoriali in aggiunta ai servizi di quartiere, se non con indici fondiari molto alti e quindi edificazione molto densa e/o in altezza.

Quindi, o si coinvolgono aree esterne agli ex scali Fs (perequandole con quote edificatorie dello 0,70 che FS pretende solo per sé) o si dovrebbe ridurre l' $\mathrm{I}_{\mathrm{t}}$ a $0,50 \mathrm{mq} / \mathrm{mq}$.

In questo senso, la vicenda del dopo Expo si connette inevitabilmente con quella dell'Accordo di Programma con FS sul riuso degli ex Scali che, se si concluderà così come è stato annunciato da Giunta e FS all'inizio di settembre, avrebbe già deciso l'80$90 \%$ del prossimo PGT (quello attuale si avvia a scadenza, con tutta l'illogicità della Legge urbanistica lombarda n. 12/2005 che non prevede alcuno strumento di indirizzo strategico di lungo periodo) senza alcune visione generale di quali altre aree potrebbero essere coinvolte in una logica di perequazione (tanto sbandierata da urbanisti e amministratori di tendenza, ma quasi mai realmente praticata), soprattutto nella localizzazione dei parchi urbani e grandi servizi territoriali, che le grandi trasformazioni urbane dovrebbero caricarsi per 15-17,5 mq/ab., oltre ai $18-26,5 \mathrm{mq} / \mathrm{ab}$. per servizi pubblici propri. In questa urbanistica à la carte che è la sommatoria di PII e Accordi di Programma (spesso incomprensibilmente intrecciati tra loro nella medesima procedura dal machiavellismo perverso della dirigenza dell'urbanistica milanese passata indenne sotto amministrazioni comunali di ogni tipo politico, quasi solo per confondere le critiche procedurali dei ricorsi amministrativi dei cittadini) comune e grandi proprietà fondiarie (oggi non più agrarie, ma per lo più aree industriali, infrastrutturali e a pubblici servizi - vedi ex caserme e/o ospedali, ecc. - dismesse o dismettibili) si trovano comunemente interessati a massimizzarne di volta in volta lo sfruttamento della rendita fondiaria derivante per finanziare gli investimenti nei propri obiettivi societari o istituzionali, mentre l'esito urbanistico-insediativo è un esito secondario residuale spesso incontrollato.

Ad esempio, nel caso dell'Accordo di Programma con Fs l'interesse comune dei sottoscrittori è rinchiudere tutto il contrattabile

Dida????

Fonte: ????????????? 
(nuovo uso immobiliare, quote di edilizia pubblica, nuovi investimenti in stazioni e linee, nuovi parchi e servizi territoriali) nell'ambito delle proprietà FS.

Ciò costringe la nuova edificazione in densità fondiarie e altezze troppo elevate, mentre con l'indice edificatorio territoriale contrattato $\left(\mathrm{I}_{\mathrm{t}}=0,70 \mathrm{mq} / \mathrm{mq}\right.$, di cui 0,35 ad edilizia libera e 0,35 ad edilizia convenzionata, ma anche quest'ultima produrrà per FS una rendita anche se forse ridotta!) per non produrre indici fondiari troppo elevati (oltre $6 \mathrm{mc} / \mathrm{mq}$ !) bisognerebbe collocare i parchi territoriali su aree di altre proprietà che si ritenesse utilmente di non far edificare a case o negozi (come, invece, oggi più d'uno propone per il dopo Expo), le quali in contraccambio acquisirebbero una quota virtuale dell'indice edificatorio delle aree Fs (presumibilmente attorno a $0,20 \mathrm{mq} /$ $\mathrm{mq})$ che può utilmente essere 'perequata' sul vasto plateau di aree pubbliche dismesse a dimensione metropolitana (a partire appunto dall'Expo in dismissione, ma anche più in generale sulla direttrice da Rho a Sesto San Giovanni).

Sull'area dell'ex Expo potrebbero allora permanentemente rimanere le funzioni di indirizzo pubblico delle politiche agroalimentari ed altre attività di interesse pubblico, un nuovo polo delle facoltà scientifiche dell'Università Statale, altre attività $\mathrm{d}$ i innovazione e ricerca, facendone il nuovo Centro Direzionale metropolitano, e non con funzioni residenziali, qui particolarmente inadatte.

Se, invece, si allarga l'orizzonte al quadro complessivo della città (che è quello che dovrebbe 'governare' il PGT, sia pur col limite intrinseco della troppo breve durata) allora l'indice $\mathrm{I}_{t}$ $=0,70 \mathrm{mq} / \mathrm{mq}$ per gli ex Scali FS può essere accettabile (pur con gli opportuni accorgimenti di assetto tipologico-distributivo o ad esempio prescrizione di parcheggi pubblici interrati o pluripiano, anziché in superficie, ciò che aumenta non poco i costi urbanizzativi degli operatori immobiliari), solo rinunciando a realizzare su quelle aree buona parte dei $15 \mathrm{mq} / \mathrm{ab}$. di parchi territoriali, e perequandole con altre o facendoli monetizzare (a prezzo di mercato, non come a Citylife pagata 2.000 euro/mq e monetizzata a 300 euro!) per poi espropriare le aree a parchi pubblici dove più opportuno; altrimenti, l'indice accettabile dovrebbe scendere verso $I_{t}=0,50 \mathrm{mq} / \mathrm{mq}$.
Senza di ciò, il prossimo PGT si troverebbe costretto in una visione monca e prefigurata dalla conclusione di accordi raggiunti su interessi parziali sia di FS sia dell'Amministrazione Comunale (e non della Città, in quanto tale). A meno che, come spesso è capitato, il nuovo PGT sia la riapertura a tutto campo di uno spazio per gli interessi particolaristici diffusi che non hanno avuto dimensione e forza per accreditarsi prima nella sommatoria casuale dei vari PII e Accordi di Programma. Purtroppo, temo sia la prospettiva più realistica e attendibile.

\section{Note}

1. «Può essere utile, a partire dall' $I_{\mathrm{e}}$, e cioè da una definita immagine dell'insediamento residenziale e della sua morfologia (pur con quella approssimazione che è rappresentata dal definire l'immagine attraverso il solo $\mathrm{I}_{\mathrm{ef}}$ ), arrivare all' $\mathrm{I}_{\mathrm{et}}$ e alla quantità complessiva di aree pubbliche connesse a quell' $\mathrm{I}_{\mathrm{el}}$ ') (Falco, 1999: 111).

2. Cfr. Brenna (2008: 105-106), cap. 7.2. «Formule di congruenza degli indici urbanistici».

3. Si vedano, ad esempio, le elaborazioni tipologiche proposte in alternativa a quelle che il Comune ha acriticamente accolto dai promotori immobiliari di Citylife e Porta Nuova, da me per l'area dell'ex Fiera di Milano e per le ex stazione Varesine e per l'intera area Garibaldi/Repubblica da Jacopo Gardella e Pierfrancesco Sacerdoti con lo schema insediativo Parco Possibile. 4. Cfr. Brenna (2010), in particolare: cap. III, «La riforma del concentrico urbano come palinsesto delle determinanti insediative delle direttrici di nord-ovest. La possibile alternativa di impianto insediativo al progetto Citylife» (77-84); «Caratteri insediativi e progettuali per l'ex scalo Farini» (8591); «Critica al masterplan di Euromilano/Rem Kolhaas per Nuova Bovisa» (92-93); «Expo 2015 come Stadtkrone urbana e regionale» (94-100); «L'ex CD di Milano: un destino realmente alternativo con un impianto ispirato dalla forma urbis» (101-118).

\section{Riferimenti bibliografici}

Brenna S., 2008, La strana disfatta dell'urbanistica pubblica. Rimini: Maggioli.

Brenna S., 2010, La Strada Lombarda, Progetti per una Milano città-madre della propria cultura insediativa. Roma: Gangemi.

Falco L., 1999, L'indice di edificabilità. Torino: UTET. 